for age according to the WHO Child Growth Standards. Mean height and weight centiles were calculated, and paired T-test used to compare measurements at admission and at yearly intervals.

Results 288 children were included, of which 137 (47.2\% were male). The median age at admission was 3 years (range 0-12 years).

Mean centiles for height and weight on admission were 16.1 and 12.7 respectively. Height and weight centiles significantly increased after 1,2 and 3 years in the programme. There was also a non-statistically significant increase at 4 to 5 years, explained by the smaller numbers of children who had been enrolled in the programme for longer periods of time. At admission, $41.8 \%$ of children were stunted. This fell to $23.9 \%$ after 1 year, $17.2 \%$ after 2 years, and $16.5 \%$ after 3 years. The greatest impact on reduction in stunting was seen in children who were admitted to the programme at age 3-4 years; and the least impact was seen in those admitted at more than 5 years old.

Conclusions Children admitted to the LTO programme show a significant increase in both height and weight centiles after 1 , 2 and 3 years. Furthermore, $60.5 \%$ of children who had stunted growth on admission showed reversal of their stunting after 3 years. This reversal of stunting was most evident in children admitted before the age of 5 years, implying that interventions are most effective if they occur at an early age.

This study provides further evidence that stunting can be reversed, and that efforts should be made to improve the nutritional status of children of all ages. It demonstrates the impact that a holistic approach to education, healthcare and childcare, working within communities and families, can have on children's growth.

\section{EFFECTS OF SCREEN TIME ON SLEEP IN CHILDREN AND ADOLESCENTS: A SYSTEMATIC REVIEW}

Sin-Chin Tan, Jenny Fraser. UK

\subsection{6/bmjpo-2021-RCPCH.47}

Background Children and teenagers are spending increasing amounts of time on screen based media; in contrast, total sleep duration has been declining in the last two decades. Researchers around the world continue to examine the association between screen time and sleep, with the last updated systematic review occurred in 2015 .

Objectives Given the extensive use of screen-based media and the high prevalence of poor sleep amongst children and teenagers, our aim was to provide an update to the previous systematic literature reviews on the effects of time spent using screens on various sleep outcomes, adopting similar criteria for inclusion.

Methods A systematic search of peer-reviewed articles was conducted using OVID MEDLINE and EMBASE. The search parameters included papers up to March 2019, with no initial cut-off date.

We included all studies that used the following inclusion criteria:

1. Cohort of children or adolescents between the age of 5 and 17 ;

2. Examined the associations between any type of screen time and sleep outcomes, including sleep timing, sleep onset latency, sleep duration, sleep quality, daytime lethargy or sleepiness or any other reported outcomes related to sleep; 3. Published in the English language.

Results 99 studies were reviewed: 80\% were cross-sectional studies, $16 \%$ were prospective studies and $3 \%$ were clinical trials. Studies included children and adolescents from countries around the world. $87 \%$ of the studies showed at least one adverse sleep outcome with screen use. 43 out of 56 (77\%) studies reported significant negative impacts on sleep outcomes with television (TV) watching; Five studies showed that the mere presence of a television set in the room itself was associated with shorter sleep duration. 49 out of 53 studies reported negative effects on sleep with the use of interactive media; Amongst all technology types, social networking sites or video games at bedtime on weekdays appeared to have the greatest negative impact on sleep duration, after adjustment for a range of potential confounders. $90 \%$ of the studies related mobile device exposure to negative sleep outcomes; Sleep disturbances or insomnia-related symptoms were measured in 13 studies, with 11 reporting significant association with the use of mobile devices. Articles that either combined multiple screens into one measure or did not specify the screen type when examining its effect on sleep outcomes were categorised into unspecified screen use; 25 out of 27 studies (93\%) found an adverse correlation with at least one sleep outcome with exposure to unspecified screens. Interestingly, parental control had a moderating effect on the relationship between bedtime and screen exposure.

Conclusions Current evidence suggests that an increased amount of screen exposure leads to adverse sleep outcome in children and adolescents. Unspecified screen use (93\%), interactive media exposure (92\%) and mobile devices use (90\%) were consistently related to poor sleep outcomes. Future research is necessary to quantify a safe amount of screen exposure and determine if there is a causality link between screen time and sleep in children and adolescents.

\section{DESIGNING A WORKABLE REFLECTIVE PRACTICE GROUP FOR THE ACUTE PAEDIATRIC TEAM}

Sarah Arthur. UK

\subsection{6/bmjpo-2021-RCPCH.48}

Background 'Acute take' is rarely synonymous with 'relaxing day at work' for a health care professional. Although many of us thoroughly enjoy the challenges, we know that the digestion of the shifts may be hard and the processing, of often complex interactions, difficult. There are high standards that we place on ourselves, and finding this energy in itself can be tough.

The terms 'wellbeing', 'resilience' and 'self-reflection' involve personal processes, often evolving over time. Whilst we build our strength and learning of these nuanced skills there is increasing evidence that a safe space and a supportive network of colleagues is paramount to a healthy work relationship.

We built facilitated time into the work schedule for our team to reflect, allowing space to help one process cases and build on their own 'emotional training'.

Objectives To establish a workable format which facilitated reflection on difficult emotional clinical events and interactions 
for our heterogenous peer group. Furthermore, to determine whether colleagues would choose to keep attending the sessions and whether participation would help emotional learning from one another.

Methods We established a fortnightly one-hour Reflective Practice Group for all trainees and consultants within the neonatal and paediatric department. The group was facilitated by a trained psychoanalytic psychotherapist, with experience of leading Balint groups. Participants were encouraged to bring a case from the past few weeks and the participants were invited to reflect openly with each other, we used the structure of Balint Groups to guide us.

Questionnaires comprising quantitative and qualitative information were collected following 10 sessions. The qualitative results were collected by one of the authors and findings reviewed for common themes.

Results From 18 responses, 94\% of responders said they would reattend and welcome further sessions. The barriers and enablers to the group are themed and summarised in table $1.77 .8 \%$ of responders felt they, in just a few sessions, had learnt from others reflections.

\begin{tabular}{ll} 
Abstract 79 Table 1 & \\
\hline Barriers to reflective practice group: & \\
\hline Theme & Awkward \\
& Forced \\
Unstructured & New \\
Positive feedback on reflective practice group: & \\
Theme & Sharing \\
& Relevant \\
& Learning from others \\
Empathy \\
Beneficial \\
Experience helps \\
Closure \\
Appreciate others \\
Leadership \\
\\
\hline
\end{tabular}

Conclusions Recurring phrases included 'awkward, forced and unstructured' but also 'relevant, beneficial and closure'. Our team clearly requires flexibility on entering the group late, eating cake at a table, allowing seniors to share comfortably and for there to be a planned case. Modification of sessions can be helpful in making the groups meaningful, relevant for training needs and less alienating. A workable solution therefore needs to be an evolving quality assured one.

A frenetic work schedule may prevent the emotional digestion of the workload and cases. A scheduled session for the team to digest the week can be extremely beneficial. In our opinion the key is to adapt the sessions to fit the group of individuals for whom it is trying to support. We now plan to measure whether the 'Monthly Digest' group has a beneficial effect on colleague reflection and well-being.

\section{IT IS THE BEST OF TIMES, IT IS THE WORST OF TIMES}

Katarina Stefkova, Danielle Leemon, Rachel Bates, Shilpa Shah. Northern Ireland, United Kingdom

\subsection{6/bmjpo-2021-RCPCH.49}

Background The year 2020 has been like no other our generation has seen. News of a devastating virus never heard of before, became an ever-present reminder of our vulnerability. Lockdown, redeployment, and social distancing were our new lingo. Faced with the challenge of working long shifts under layers of personal protective equipment we needed more than ever before to look at ways of supporting and appreciating each other. That is when the 'Happiness champions' were born.

Objectives To improve staff morale, recognise and reward small or big acts of kindness, team spirit and dedication while serving in the front line during the current pandemic. All staff (see below in results) within the Children and Young people's service in Craigavon Hospital were included.

Methods A core team of 'Happiness champions' including 3 paediatric trainees and 1 consultant was created.

Laminated cards with cartoon heroes were created, with the phrase 'you are amazing, keep it up, that is all' printed on the back. Small tokens were also included in the prize.

An email portal and a whatsapp group of all staff members in paediatrics were used to promote the initiative and receive nominations each week. The nomination could be for any member within the children and young person's department irrespective of grade or role. The person nominating needed to state the reason for nomination to enable the core team to decide the winner each week.

Each week, one 'Hero of the week' was announced through the whatsapp group with a summary of the reasons for nomination along with a photo of our Hero. This was also broadcast on social media platforms (after consent) to have a further reach.

Results 17 'Heroes' were awarded, one for each week of the lockdown.

Members of staff of all levels and areas within paediatrics were nominated (nursing, allied health, administrative, domestics and medical) showing how much positive regard there was despite tough times.

$100 \%$ of our 'Heroes' were happy to receive the award and felt proud to be members of our team. This is evidenced by their wide smiles on photographs, although some may be hidden behind surgical masks. There was overwhelming response on social media with over a thousand likes and hundreds of shares, further spreading our message of happiness.

Conclusions The worst of times are only an opportunity to create the best of times.

Staff morale, resilience and team spirit greatly improved with this weekly feature.

Creating a virtuous cycle can be easy, fun and immensely satisfying.

We couldn't have said it better than Albus Dumbledore 'Happiness can be found even in the darkest of times, if only one remembers to turn on the light' 